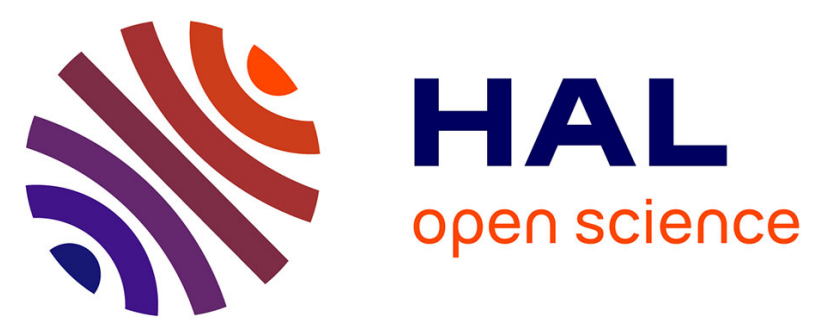

\title{
Coherence measurements and coherent diffractive imaging at FLASH
}

* I A Vartanyants, a P Mancuso, A Singer, O M Yefanov, \& J Gulden

\section{To cite this version:}

* I A Vartanyants, a P Mancuso, A Singer, O M Yefanov, \& J Gulden. Coherence measurements and coherent diffractive imaging at FLASH. Journal of Physics B: Atomic, Molecular and Optical Physics, 2010, 43 (19), pp.194016. 10.1088/0953-4075/43/19/194016 . hal-00569849

\section{HAL Id: hal-00569849 \\ https://hal.science/hal-00569849}

Submitted on 25 Feb 2011

HAL is a multi-disciplinary open access archive for the deposit and dissemination of scientific research documents, whether they are published or not. The documents may come from teaching and research institutions in France or abroad, or from public or private research centers.
L'archive ouverte pluridisciplinaire HAL, est destinée au dépôt et à la diffusion de documents scientifiques de niveau recherche, publiés ou non, émanant des établissements d'enseignement et de recherche français ou étrangers, des laboratoires publics ou privés. 


\title{
Coherence Measurements and Coherent Diffractive Imaging at FLASH
}

\author{
I. A. Vartanyants*, A. P. Mancuso, A. Singer, O. M. Yefanov, \& J. Gulden \\ Deutsches Elektronen-Synchrotron DESY, Notkestraße 85, D-22607 Hamburg, Germany
}

\section{June 2, 2010}

\begin{abstract}
We present an overview of recent experiments performed at the free-electron laser (FEL) FLASH at DESY in Hamburg. Experiments were focused on coherence measurements and coherent x-ray diffractive imaging (CXDI) of periodic and non-periodic biological samples. Young's double slit experiment was performed at FLASH to measure its coherence properties at a fundamental wavelength of $13.7 \mathrm{~nm}$. Additionally, a uniformly redundant array (URA) was used to measure coherence properties of the $3^{\text {rd }}$ harmonic $(2.7 \mathrm{~nm})$ of an $8 \mathrm{~nm}$ fundamental wavelength at FLASH. Coherent imaging of a two-dimensional (2D) finite crystal structure using a single pulse train of FLASH radiation was demonstrated. We show that the structure is reconstructed to the detector limited resolution of $220 \mathrm{~nm}$, given an adequate signal to noise ratio. We have also employed CXDI in a nondestructive regime to compare images of a biological sample using single, femtosecond pulses of FLASH radiation. We have verified that images reconstructed using CXDI are similar for statistically different FEL pulses. We have also demonstrated Fourier transform holography (FTH) of the same biological sample and present diffraction data measured at the third harmonic of FLASH, reaching into the water window.
\end{abstract}

PACS: $41.60 . \mathrm{Cr}, 42.25 . \mathrm{Fx}, 42.25 . \mathrm{Kb}, 42.30 . \mathrm{Rx}, 87.59 .-\mathrm{e}$

Keywords: free electron laser, coherent imaging, phase retrieval, biological imaging

* Corresponding author. Email: ivan.vartaniants@desy.de 


\section{Introduction}

The invention of optical lasers in the 1960s has dramatically changed the scope of scientific research. New directions in atomic, optical and laser-matter interaction physics, based on the use of the unique properties of laser radiation, have emerged and become mature in subsequent years. One of the key challenges was to create laser radiation at shorter wavelengths. It quickly became clear that lasers at $\mathrm{x}$-ray wavelengths (on the order of $0.1 \mathrm{~nm}$ ) can not be built using the same physical principles as those at optical wavelengths. This is due in part to the difficulty of producing highly efficient resonator cavities at these wavelengths. In the 1980s it was suggested to create laser type radiation at short wavelengths from linear accelerators using the Self Amplified Spontaneous Emission (SASE) process ([1] and references therein). This alternative route is very attractive due to the unique properties of radiation at X-ray wavelengths combined with high peak brightness (10 orders of magnitude higher than any existing x-ray source), a high degree of coherence and ultra-short pulse duration (below $100 \mathrm{fs}$ ). With the start of operation of the first XUV free-electron laser (FEL) FLASH [2] at DESY in Hamburg these theoretical predictions were experimentally confirmed.

One of the major challenges in modern life sciences is to reveal the structure of protein molecules, which is a prerequisite for understanding the function of larger biological complexes. At the forefront of modern structural investigations is the knowledge of the functionality of biological systems on different length and time scales. The key progress in solving the structure of proteins in past decades was due to the development of phasing methods [3] which allow the determination of the structure of complex molecules that crystallize. In spite of considerable progress in macromolecular crystallography, crystallization and radiation damage are still bottlenecks in protein structure determination.

Conventional x-ray imaging of biological specimens at synchrotron sources [4] suffers from radiation damage, which limits the resolution of the resultant images [5]. Breaking this resolution limit may be possible by the use of ultrabright, ultrashort pulses from FELs. If these pulses are short enough they may scatter from the specimen before it is destroyed by this pulse $[6,7]$. Structural information from the undamaged sample will be measured in its diffraction pattern. To produce an image of the object at a resolution higher than that typically measured at conventional $\mathrm{x}$-ray sources, techniques such as coherent $\mathrm{x}$-ray diffractive imaging (CXDI) may be used.

The basic idea of coherent imaging developed by several groups $[8,9,10,11,12]$ involves a finite sized object being illuminated with coherent radiation (Fig. 1). Its corresponding far-field diffraction 
pattern is then measured, and importantly, sampled sufficiently. Given the knowledge of the object's finite extent, known as its finite support, the measured diffraction pattern can be inverted uniquely ${ }^{1}$ [13] using phase retrieval methods $[14,15,16]$ to reconstruct an image of the object. The advantages here are the absence of imaging optics required to make the measurement, and that the possible resolution is limited, in principle, only by diffraction, and in practice only by the signal-to-noise ratio in the diffraction measurement. This freedom from the resolution restrictions of conventional x-ray microscopy becomes increasingly important as the length scale of samples studied decreases.

In addition to CXDI there are other coherent imaging techniques such as digital in-line holography $[18,19,20]$ and Fourier Transform Holography (FTH) $[21,22,23]$ that are based on using a reference wave that originates from a pinhole or scatterer located either in front of the sample (in-line holography), or in the plane of the sample (FTH). As a result, the phase problem can be solved without ambiguity, but with additional restrictions on spatial resolution imposed by the generation of the reference wave and the commensurate reduction of the incident beam intensity.

Free-electron lasers are particularly well suited for coherent imaging (see for a review of FLASH coherent imaging experiments [24]). They provide femtosecond coherent pulses with extremely high power. The combination of these unique properties could allow the realization of coherent X-ray imaging on biological systems (see for a review of biological imaging at synchrotron and FEL sources [25]). FLASH, providing up to $10^{13} \mathrm{XUV}$-coherent photons in a single pulse of tens of femtoseconds, is a unique source for coherent imaging applications. The first demonstrations of single pulse and time-resolved CXDI experiments were performed recently at FLASH [26,27,28].

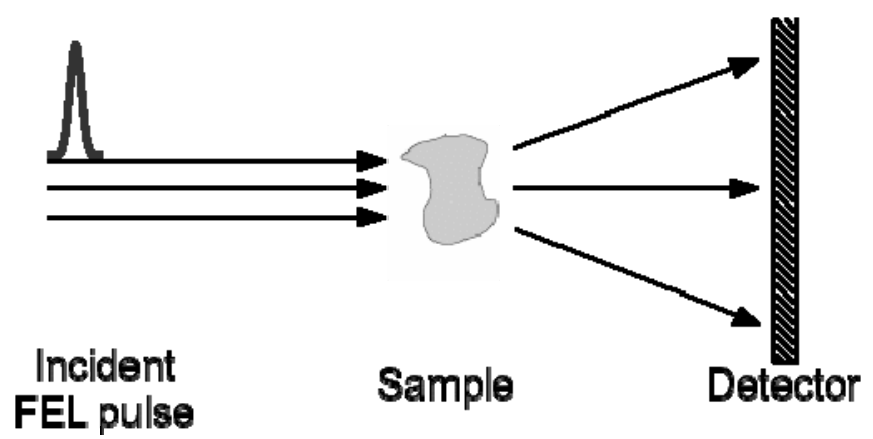

Figure 1. Schematic of a coherent $x$-ray diffractive imaging experiment. The incident radiation, in this case a single FEL pulse, illuminates the sample from the left. The scattered radiation propagates to an area detector where the diffracted intensities are measured.

\footnotetext{
${ }^{1}$ We do not consider here trivial solutions such as shifts of the image and its complex conjugate as different solutions. We also note that phase retrieval with a support constraint is more stable in higher (two- or three-) dimensions [17].
} 
For all these coherence based imaging techniques highly coherent incident radiation is necessary and we start our overview (section 2) with the results of our coherence measurements performed at FLASH. We continue in section 3 by presenting coherent pulse two-dimensional (2D) crystallography and then proceed in section 4 with single pulse coherent imaging of non-reproducible biological samples at FLASH. We complete this review with conclusions and an outlook.

\section{Coherence at FLASH}

Coherence is one of the principal features of laser sources. The degree of coherence describes the strength of correlations in a wavefield between different points in space and time. The measurement of the coherence properties of existing FELs is of vital importance for understanding the physical principles that describe the SASE generation of coherent beams, for optimization of the parameters of these sources, and for the construction of instruments to exploit them. These measurements are also important for planning future experiments that utilize the coherence properties of high power FEL beams, for example, for different applications in materials science [29] and biology, including possibilities such as single molecule imaging [6,7]. Young's double slit experiment is one of the most efficient and widely used methods for measuring the transverse coherence properties of wavefields and we have used it to analyze the transverse coherence properties of FLASH [30].

In the theory of coherence, second order correlations of wavefields are described [31,32] by the mutual coherence function (MCF) $\Gamma\left(\mathbf{r}_{1}, \mathbf{r}_{2}, \tau\right)$, that defines the correlations between two complex values of the electric field $E\left(\mathbf{r}_{1}, \mathrm{t}\right)$ and $E\left(\mathbf{r}_{2}, \mathrm{t}+\tau\right)$ at different points $\mathbf{r}_{1}$ and $\mathbf{r}_{2}$ and separated by the time interval $\tau$

$$
\Gamma\left(\mathbf{r}_{1}, \mathbf{r}_{2}, \tau\right)=\left\langle E\left(\mathbf{r}_{1}, \mathrm{t}\right) E^{*}\left(\mathbf{r}_{2}, \mathrm{t}+\tau\right)\right\rangle
$$

where the brackets $\langle\ldots\rangle$ indicate the ensemble average. The normalized function of $\Gamma\left(\mathbf{r}_{1}, \mathbf{r}_{2}, \tau\right)$ is called the complex degree of coherence

$$
\gamma_{12}(\tau)=\Gamma\left(\mathbf{r}_{1}, \mathbf{r}_{2}, \tau\right) / I\left(\mathbf{r}_{1}\right)^{1 / 2} I\left(\mathbf{r}_{2}\right)^{1 / 2}
$$

where $I\left(\mathbf{r}_{1}\right)$ and $I\left(\mathbf{r}_{2}\right)$ are the averaged intensities at points $\mathbf{r}_{1}$ and $\mathbf{r}_{2}$. The modulus of $\gamma_{12}(\tau)$ is directly measured from the visibility of fringes in Young's double slit experiment. 
a). Measurements of transverse coherence at FLASH at the fundamental wavelength

The transverse coherence was measured using a double slit experiment (see Fig. 2 (a)) at a fundamental wavelength of $13.7 \mathrm{~nm}$ during the FLASH commissioning phase. The experiment was performed with a set of horizontal and vertical slits positioned a distance of $20 \mathrm{~m}$ downstream from the last operating undulator module in the FEL tunnel. The distance $d$ between the slit centers was 150, 300 and $600 \mu \mathrm{m}$ for both vertical and horizontal slit pairs. The individual slit width was $30 \mu \mathrm{m}$ for the first two pairs and $50 \mu \mathrm{m}$ for the last pair. The detector was located at a distance of $4.44 \mathrm{~m}$ downstream from the slit mask. Each interference pattern measured in this experiment was a result of the accumulation of ten bunches in a single train of FEL radiation and each measurement for a given slit separation was repeated ten times. A typical interference pattern measured on the detector with a horizontal slit separation of $150 \mu \mathrm{m}$ is shown in Fig. 2 (b).

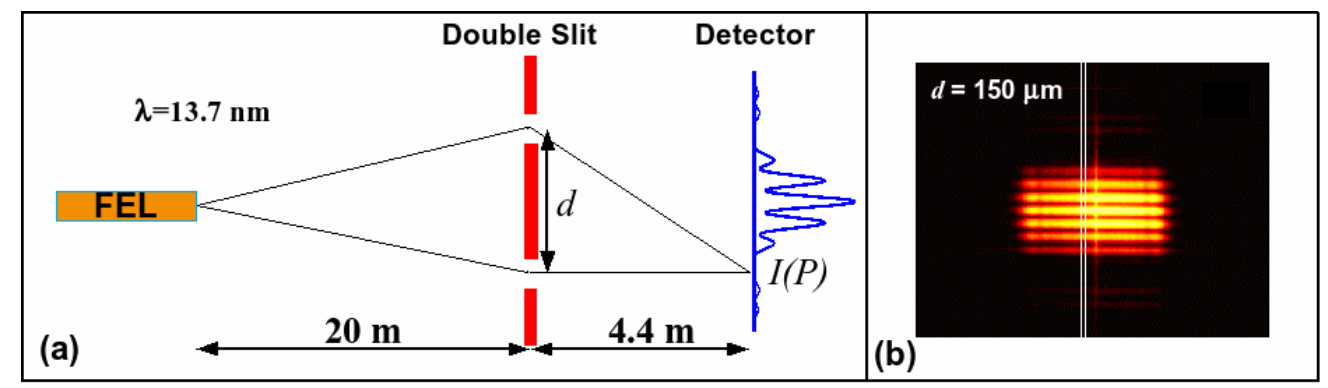

Figure 2 (Colour Online) (a) Schematic of the double slit experiment. (b) A typical experimental interference pattern.

A fit to each series of experimental data was performed with the following expression, which is valid for a narrow bandwidth radiation [31]

$$
I(P)=I_{1}(P)+I_{2}(P)+2 \sqrt{I_{1}(P) I_{2}(P)}\left|\gamma_{12}\right| \cos \left[\omega \tau-\alpha_{12}\right],
$$

where $I_{1}(\mathrm{P})$ and $I_{2}(\mathrm{P})$ are intensities at the detector position $\mathrm{P}$ corresponding to propagation of radiation from each of the slits separately, $\tau$ is the time delay for the radiation to reach point $\mathrm{P}$ at the detector from slits one and two and $\alpha_{12}(\tau)$ is the relative phase. Typical results of the fit for different slit separations are shown in Fig. 3(a,b). As a result of the fitting, the absolute value of the complex coherence factor $\left|\gamma_{12}\right|$ was determined for each slit separation in both the horizontal and vertical directions (see Fig. 3 (c)). 

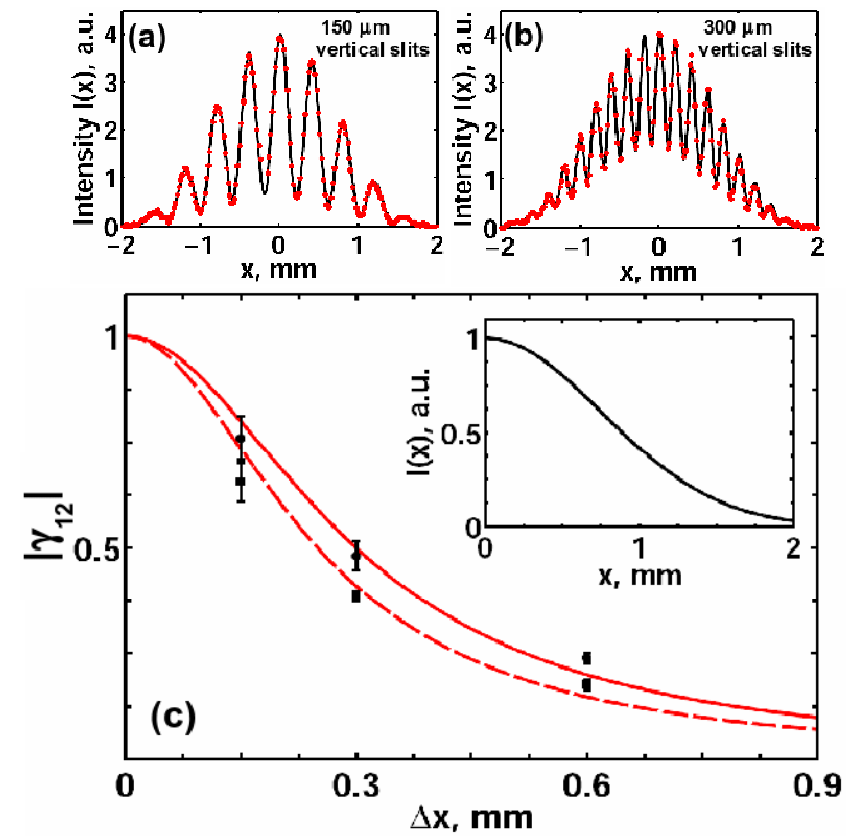

Figure 3. (Colour Online) (a),(b) Results of the fit (solid lines) to experimental data (dots) for different slit separation in the horizontal direction. (c) Modulus of the complex degree of coherence as a function of slit separation in the horizontal (circles) and vertical (squares) directions determined from the fit to the experimental data. A Lorentzian fit to $\left|\gamma_{12}(\Delta x)\right|$ is shown by the solid (vertical direction) and dashed (horizontal direction) lines. The intensity distribution is shown in the inset. Images and figures from [30].

Close inspection of the results (Fig. 3) shows that the radiation field is clearly not fully coherent, but is rather reduced to a few hundred microns length scale. The magnitude of the degree of coherence can be approximated by a Lorentzian function with coherence lengths $l_{\mathrm{c}}(\mathrm{H})=300 \mu \mathrm{m}$ in the horizontal and $l_{\mathrm{c}}(\mathrm{V})=250 \mu \mathrm{m}$ in the vertical directions. The results are in very good agreement with the apparent source size observed with a wave front sensor at similar operating conditions of FLASH. The source size was about 2.5 times larger than reported in [2], due to the FEL not operating at full saturation during these measurements.

b). Measurements of the transverse coherence of a $3^{\text {rd }}$ harmonic at FLASH

In the previous section we described a classical Young's double slit experiment performed at FLASH. This allowed us to measure the transverse coherence at certain relative distances, which correspond to the slit separations. A set of slits with different slit separations was necessary to measure the full shape of $\left|\gamma_{12}\right|$. In contrast to these experiments, measurements of coherence using uniformly redundant arrays (URA) [33] provide the value of the complex degree of coherence for a range of 
relative distances in a single measurement. A URA aperture consists of many slits, which are arranged in such a way that on a finite grid each slit separation is present an equal number of times. Due to the nature of the SASE process [1] each pulse from the FEL is statistically different. Averaging over different pulses may lead to a reduction of the measured value of the coherence, hence the measurements of the coherence properties of single pulses are of high importance.

The diffraction pattern of an aperture (a URA in our case) illuminated by partially coherent, narrow band light can be written as a convolution of the Fourier transform of the complex degree of coherence $\gamma(\mathbf{q})$ and the diffraction pattern produced by fully coherent radiation $I_{\operatorname{coh}}(\mathbf{q})[34,35]$

$$
I(\mathbf{q})=\gamma(\mathbf{q}) \otimes I_{\mathrm{coh}}(\mathbf{q})
$$

where $\otimes$ denotes the convolution operation. The Fourier transform of this expression gives the values of the complex degree of coherence $\gamma_{12}(\Delta \mathbf{r})$ for different separations of points $\Delta \mathbf{r}=\mathbf{r}_{2}-\mathbf{r}_{1}$

$$
\gamma_{12}(\Delta \mathbf{r})=I(\Delta \mathbf{r}) / I_{\operatorname{coh}}(\Delta \mathbf{r})
$$

where $I_{\mathrm{coh}}(\Delta \mathbf{r})$ reduces to the autocorrelation function of an aperture in the far-field approximation with plane wave illumination.

According to Eq. (5), the knowledge of $I_{\mathrm{coh}}(\Delta \mathbf{r})$ and the measurement of the diffraction pattern produced by a partially coherent beam provides a way to obtain the complex degree of coherence $\gamma_{12}(\Delta \mathbf{r})$ of the radiation at the aperture. To optimize this measurement the aperture can be refined to find the best functional form of the autocorrelation function. It can be shown [36] that in the far-field, given a known incident wavefront, a measurement with a URA as the aperture is an exemplary tool for diffraction based coherence measurements. It follows directly from Eq. (5) that the complex degree of coherence can be determined for all relative distances up to the size of the URA aperture in a single measurement. 

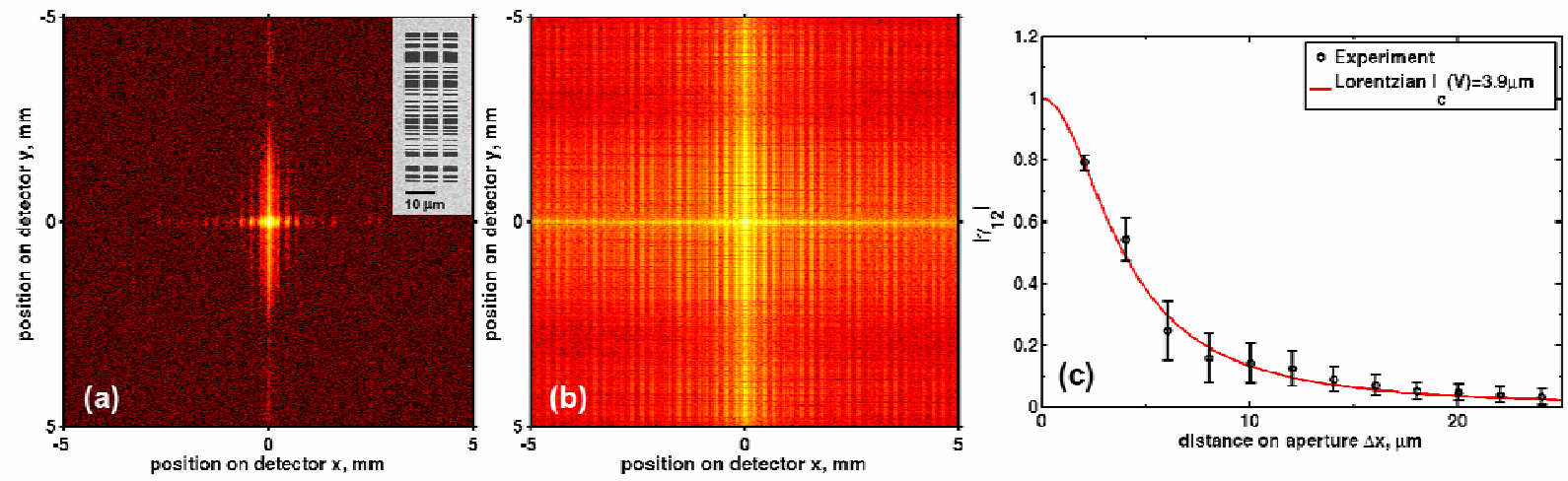

Figure 4. (Colour Online) (a) Average diffraction pattern measured in the vertical direction with the URA from 10 FEL pulses. SEM image of the URA is shown in the inset. (b) The diffraction pattern $I_{\text {coh }}(q)$ calculated in the Fresnel approximation for the URA shown in (a). (c) The modulus of the complex degree of coherence $\left|\gamma_{12}\right|$ (black circles) determined from the analysis of the set of 30 diffraction patterns containing 10 pulses each. Error bars are determined as a standard deviation for this set of experimental data. A Lorenzian fit (red line) gives the value $l_{c}(V)=3.9 \mu \mathrm{m}$ for the coherence length in the vertical direction.

Coherence measurements with URAs were performed at the plane grating monochromator beamline PG2 [37] at FLASH where we measured the transverse coherence of the $3^{\text {rd }}$ harmonic (wavelength $2.7 \mathrm{~nm}$ ) of a fundamental wavelength $8 \mathrm{~nm}$ [38]. The URA structure was manufactured on a $100 \mathrm{~nm}$ thick $\mathrm{Si}_{3} \mathrm{Ni}_{4}$ membrane coated with $600 \mathrm{~nm}$ of gold and $200 \mathrm{~nm}$ of palladium by Focused Ion Beam (FIB) milling (see inset in Fig. 4 (a)). The dimensions of the aperture were $50.5 \times 18 \mu \mathrm{m}^{2}$. Two identical URAs were manufactured and positioned on the sample holder to measure the transverse coherence in the vertical and horizontal directions. The URAs were positioned in the $300 \times 50 \mu \mathrm{m}^{2}$ focus of the beam (elongated in the vertical direction due to the use of a horizontal plane grating) and the diffracted radiation was detected using a CCD camera $0.53 \mathrm{~m}$ downstream from the sample. Thirty diffraction patterns with an exposure time of $2 \mathrm{~s}$ containing 10 pulses each were collected for horizontal and vertical directions. An averaged diffraction pattern consisting of 10 pulses for the vertical orientation of the URA is shown in Fig. 4(a). Expression (5) was used to evaluate the experimental data. An aperture mask (Fig. 4 (a)) was used to calculate $I_{\text {coh }}(\Delta \mathbf{r})$ in the Fresnel approximation (Fig. 4(b)) for a plane wave illumination of the aperture. The modulus of the complex degree of coherence was evaluated as described earlier for each set of 10 pulses. Result of this evaluation is shown in Fig. 4(c). In this specific case of multiple pulse measurements, we estimate the average transverse coherence length to be $3.9 \pm 0.8 \mu \mathrm{m}$ in the vertical direction and $5.9 \pm 0.9 \mu \mathrm{m}$ in the horizontal direction. 
c) Mode expansion for coherence characterization

To describe the coherence properties of FLASH in a statistical optics framework, we perform a mode expansion of the correlation functions that describe the FEL wavefield [30]. Correlations of the field in the spatial-frequency domain are determined by the cross-spectral density function (CSD) $W\left(\mathbf{r}_{1}\right.$, $\left.\mathbf{r}_{2}, \omega\right)$, which is a Fourier transform of the $\operatorname{MCF} \Gamma\left(\mathbf{r}_{1}, \mathbf{r}_{2}, \tau\right)(1)$

$W\left(\mathbf{r}_{1}, \mathbf{r}_{2}, \omega\right)=\int \Gamma\left(\mathbf{r}_{1}, \mathbf{r}_{2}, \tau\right) \mathrm{e}^{-i \omega \tau} \mathrm{d} \tau$

The spectral density of the field $S(\mathbf{r}, \omega)$ is defined as the CSD function taken at the same position $S(\mathbf{r}, \omega)=\mathrm{W}(\mathbf{r}, \mathbf{r}, \omega)$. The normalized version of the CSD $W\left(\mathbf{r}_{1}, \mathbf{r}_{2}, \omega\right)$ is the spectral degree of coherence

$$
\mu\left(\mathbf{r}_{1}, \mathbf{r}_{2}, \omega\right)=W\left(\mathbf{r}_{1}, \mathbf{r}_{2}, \omega\right) / S^{1 / 2}\left(\mathbf{r}_{1}, \omega\right) S^{1 / 2}\left(\mathbf{r}_{2}, \omega\right)
$$

It has been shown [32], that under very general conditions, one can represent the CSD of a partially coherent statistically stationary field of any state of coherence as a sum of independent coherent modes

$W\left(\mathbf{r}_{1}, \mathbf{r}_{2},\right)=\sum_{\mathrm{j}} \beta_{\mathrm{j}} E_{\mathrm{j}}^{*}\left(\mathbf{r}_{1}\right) E_{\mathrm{j}}\left(\mathbf{r}_{2}\right)$,

where $\beta_{j}$ and $E_{j}\left(\mathbf{r}_{2}\right)$ are respectively the eigenvalues and eigenfunctions of the Fredholm integral equation

$\int W\left(\mathbf{r}_{1}, \mathbf{r}_{2},\right) E_{\mathrm{j}}\left(\mathbf{r}_{1}\right)=\beta_{\mathrm{j}} E_{\mathrm{j}}\left(\mathbf{r}_{2}\right)$.

For our purposes it is especially important to calculate correlation functions at different distances from the source. These values of the CSD can be obtained by propagation of the individual coherent modes. Due to statistical independence of the modes the CSD, after propagating a distance $\mathrm{z}$, is given as a sum of propagated modes $E_{j}(\mathbf{r}, \mathrm{z})$ with the same eigenvalues $\beta_{j}$

$$
W\left(\mathbf{r}_{1}, \mathbf{r}_{2}, \mathrm{z}\right)=\sum_{\mathrm{j}} \beta_{j} E_{j} *\left(\mathbf{r}_{1}, \mathrm{z}\right) E_{j}\left(\mathbf{r}_{2}, \mathrm{z}\right) .
$$


We have used this approach to make a realistic and simple estimate of the coherence properties of the existing FLASH source. We used a Gaussian Schell-model (GSM) [32] to describe statistical properties of radiation from FLASH source. For these calculations we have used the parameters of FLASH as reported in [2] for a fundamental wavelength of $13.7 \mathrm{~nm}$. The CSD was calculated at a distance $20 \mathrm{~m}$ downstream from the source using Eq. (10) including seven modes. In Fig. 5 the results of these calculations are presented. An analysis of these results shows that, for the parameters of FLASH, a small number of transverse modes contribute to the total field (Fig. 5c) that reduces the degree of coherence of the FEL source [39]. The second mode is about $40 \%$ of the fundamental, and the contribution of the sixth mode is more than two orders of magnitude smaller than the fundamental. The contribution of each mode to the spectral degree of coherence is shown in Fig. 6. An analysis of the results also gives a theoretical estimate of the upper value of the transverse coherence $l_{\mathrm{c}}=715 \mu \mathrm{m}$ at a distance of $20 \mathrm{~m}$ downstream from FLASH.
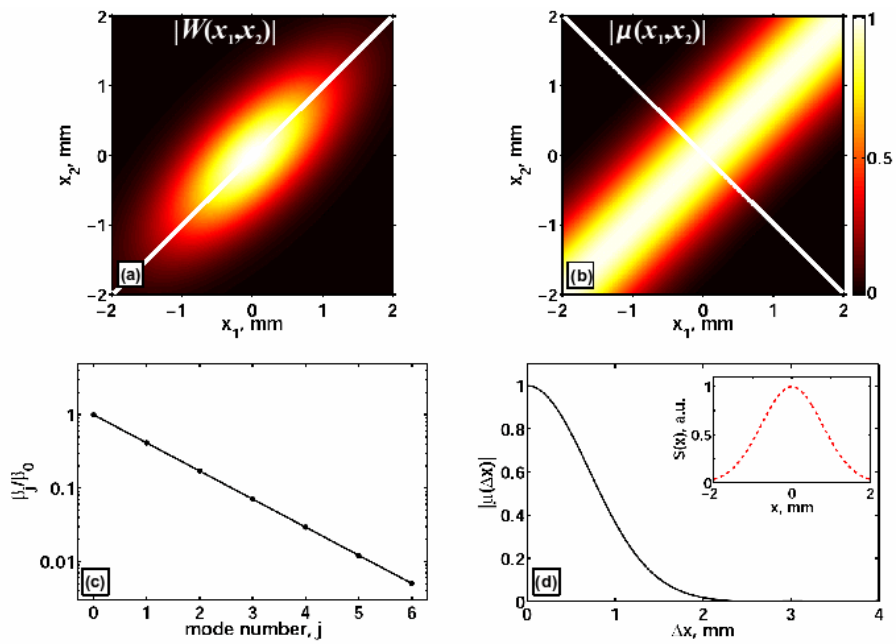

Figure 5. (Colour Online) (a) Modulus of the cross spectral density $\left|W\left(x_{1}, x_{2}\right)\right|$. (b) Modulus of the spectral degree of coherence $\left|\mu\left(x_{1}, x_{2}\right)\right|$. (c) The ratio $\beta_{j} / \beta_{0}$ of the eigenvalue $\beta_{j}$ to the lowest order eigenvalue $\beta_{0}$ as a function of mode number $j$. (d) Modulus of the spectral degree of transverse coherence $|\mu(\Delta \mathbf{x})|$ taken along the white line in (b). In the inset the spectral density $S(x)$ is shown taken along the white line in (a). Images and figures from [30]. 

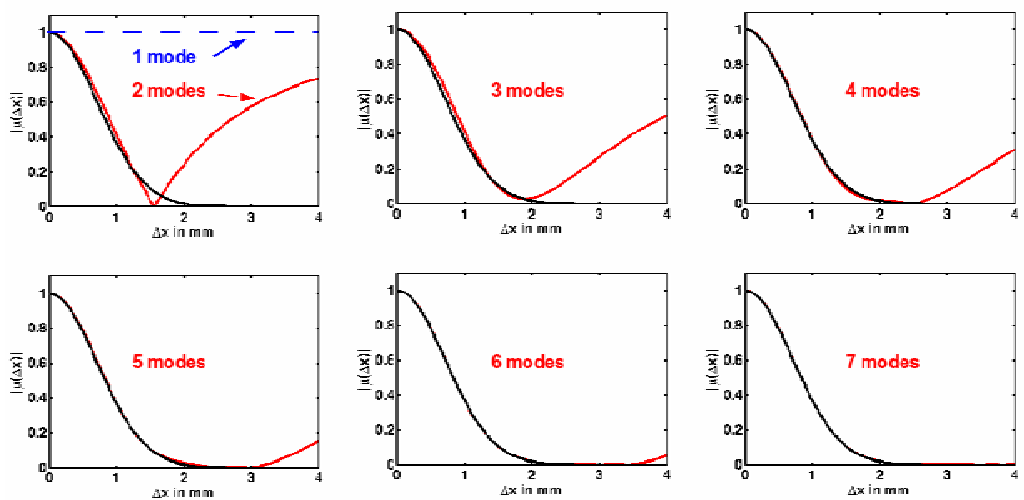

Figure 6. (Colour Online) Contribution of individual modes to the modulus of the degree of coherence. Seven modes are sufficient to describe the coherence properties of FLASH.

\section{Coherent-Pulse 2D Crystallography at FLASH}

One of the most exciting proposals to make use of these ultrabright, highly coherent FEL pulses is to determine the $3 \mathrm{D}$ structure of single biological molecules to sub-nanometer resolution $[6,7]$, which is a resolution beyond the conventional radiation damage limit. A single molecule, however, can produce only a very weak signal from even the brightest of FEL pulses [25] (see Fig. 7(a)). One idea is based on imaging many copies of reproducible biological samples, which are injected into the ultrashort pulses of the FEL beam in a random orientation (see Fig. 7(a)). By classifying and orienting many 2D diffraction patterns, a single three-dimensional diffraction pattern may be composed and reconstructed to give a 3D representation of the sample investigated. Recent simulations suggest that this method of interpreting the data is feasible [40,41]. In particular, new algorithms have been developed $[42,43]$ that explicitly address low photon counts in individual 2D diffraction patterns. Another way to circumvent this limitation is to use $2 \mathrm{D}$ or $3 \mathrm{D}$ periodic sample arrays to increase the signal diffracted from each unit for a given single pulse photon flux. Membrane proteins are a particular example of proteins that more readily form two-dimensional crystals rather than three dimensional crystals [44]. 

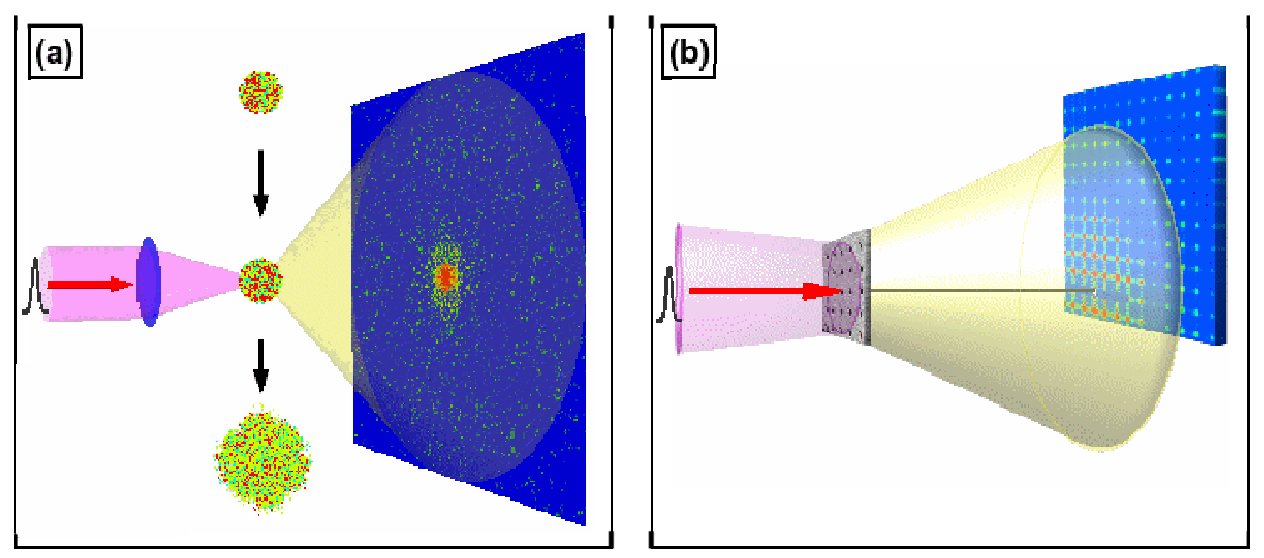

Figure. 7. (Colour Online) (a) Schematic image of virus particles injected into a focused FEL beam and its diffraction pattern produced from a single pulse FEL radiation. The virus explodes after the pulse has interacted with it. Note the low photon count in a diffraction pattern produced by a single pulse of $10^{12}$ photons focused to a $100 \mathrm{~nm}$ spot size. (b) Schematic of coherent diffraction from a 2D crystal.

We show 2D finite crystallography [45] by using an artificial 2D crystal that was manufactured with FIB milling to produce 5 x 5 units of repeated larger, $500 \mathrm{~nm}$ diameter apertures (representing a 'heavy' atom in conventional crystallography) and smaller, $200 \mathrm{~nm}$ diameter apertures (representing a 'light' atom) features in a gold palladium substrate. This structure was illuminated with one single pulse train of FEL radiation at FLASH.
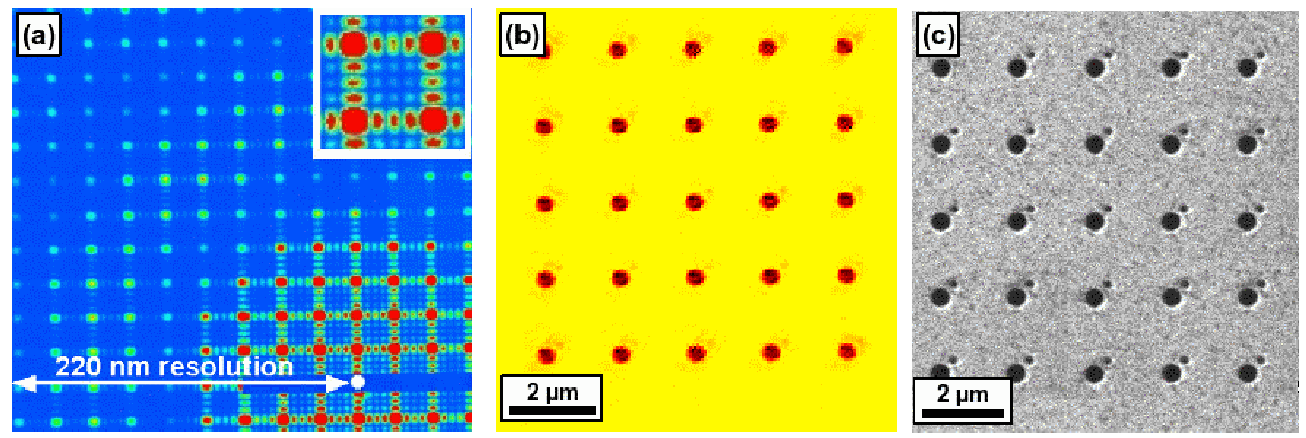

Figure 8. (Colour Online) (a) Far-field diffraction data measured from a single train of 21 pulses from the FEL. The white dot indicates the center of the incoming beam. (Inset) Enlarged region of diffraction pattern. (b) The final reconstructed image. (c) Scanning Ion Micrograph (SIM) of the manufactured sample. Images and figures from [45].

The diffraction data was measured at FLASH on the PG2 monochromator beamline with a fundamental wavelength of $7.97 \mathrm{~nm}$. The schematic of the experiment is shown in Fig. 7(b). As incident radiation, we used a pulse train consisting of 21 pulses each of approximately a few tens of femtoseconds duration. A $0.2 \mathrm{~s}$ exposure time was used to collect a series of single pulse train data from our sample with a coherent flux on the sample area of $1.5 \times 10^{10}$ photons per train due to the beamline transmission and the overilluminated sample size. A typical single train data set is shown in Fig. 8(a). 
The diffraction pattern as measured contains signal up to the edge of the detector, corresponding to a feature size of $220 \mathrm{~nm}$ (Fig. 8). We note that the expected features of a finite, crystalline structure are observed. Bragg peaks, due to the periodicity of the array, are easily visible, as are the oscillations between the Bragg peaks resulting from the finite extent and coherent illumination of our sample. Furthermore, the form factor of the individual elements, that is the large holes, is observed as a radial intensity modulation across the pattern produced.

The preprocessed data set was reconstructed with the HIO phase retrieval algorithm [14]. Initial reconstructions demonstrated a limited resolution and a sensitivity to noise. One reason for this is that

the measured diffraction pattern has two equivalent, symmetric solutions. Due to this symmetry and the noise level in the data the reconstruction algorithm does not fully converge, but rather stagnates with two equivalent solutions superimposed over each other. To ameliorate this problem we binned the data $5 \times 5$ (for a sampling rate of 6 in each dimension). Furthermore, instead of using a large square support we applied a tighter support of 25 rectangular boxes each centered on the positions of the unit cell. By improving the signal-to-noise ratio and reducing the symmetry in real space we were able to improve the reconstruction and resolve the smallest features present in our sample (Fig. 8b). We find that the resolution in real space was better than $238 \mathrm{~nm}$, comparing favorably with our measured maximum momentum transfer that corresponds to $220 \mathrm{~nm}$ resolution (see Fig. 8 (a)).

\section{Single pulse coherent imaging of non-reproducible biological samples at FLASH}

One of the advantages of coherent imaging techniques, as described earlier, is the ability to image non-crystalline structures, thereby circumventing the need for crystallization. For the case of composing an image from multiple reproducible samples, each imaged with different FEL pulses, a prerequisite is that each FEL pulse is similar, or is characterised such that a 3D diffraction pattern can be composed from many different 2D measurements. Here we report on CXDI experiments [46] performed with femtosecond coherent pulses from FLASH on a critical point dried non-crystalline, non-reproducible biological object supported on a silicon nitride membrane. In particular, we image a diatom with statistically different SASE FEL pulses in a non-destructive regime and compare the reconstructions obtained from these different FEL pulses. 


\section{a) CXDI at fundamental wavelength}

We performed a coherent imaging experiment at FLASH, which was tuned to produce a fundamental photon wavelength of $8 \mathrm{~nm}$ and delivered to the PG2 monochromator beamline. This beamline allows access to higher harmonic FEL radiation and therefore the possibility to perform experiments in the water window. FLASH produced pulses of about 10 fs duration with 15-20 $\mu \mathrm{J}$ power per pulse in the fundamental. We recorded diffraction data from single pulses of the fundamental FEL radiation scattered from a diatom, Navicula perminuta. Diatoms are unicellular algae in which the protoplast is encased in a silica cell wall. An average of $9 \times 10^{9}$ photons per pulse (estimated by ray tracing) were delivered to a focal spot of $50 \mu \mathrm{m}$ FWHM in a dedicated vacuum chamber. About $2.5 \times 10^{8}$ photons per average pulse were then incident on the sample of $10 \times 5 \mu \mathrm{m}^{2}$ size. Fifty different single pulse diffraction patterns were recorded from the same diatom. A typical diffraction pattern collected using a single pulse is shown in Fig. 9(a). Additionally a series of ten multiple pulse measurements, each of $30 \mathrm{~s}$ duration, was recorded. The sum of these exposures (1500 pulses in total) is shown in Fig. 9(b). Note that the difference in signal between these data sets determines the resolution of the reconstructed image [45].

In Fig. 10(a-c) we show CXDI reconstructions from different single pulse measurements, while Fig. 10(d) shows a reconstruction from the integrated measurement. The reconstructions in Fig. 10 were performed by applying the GHIO [47] algorithm. It is known that, due to the SASE process [1], individual femtosecond pulses are statistically different. By comparing results for the same sample illuminated by different single pulses (Fig. 10(a-c)) we observe the same features imaged at different resolutions, dependent on the pulse intensity. This demonstrates that the statistical pulse to pulse variations of FLASH are not a limitation for coherent imaging to the resolutions achieved here. The reconstruction from the integrated measurement due to a higher flux density clearly provides the most detailed information about the diatom. 

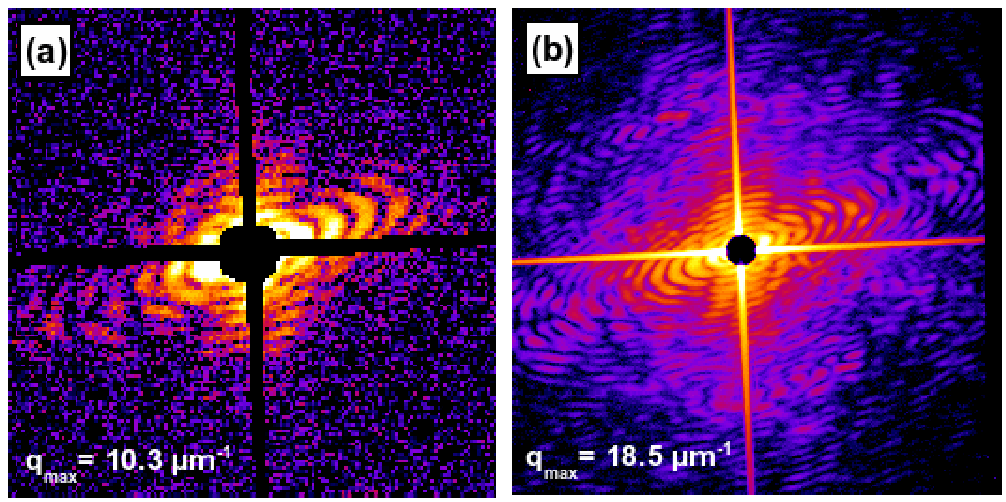

Figure 9. (Colour Online) Results of the CXDI experiment. (a) A typical single pulse diffraction pattern measured at fundamental $8 \mathrm{~nm}$ wavelength with streaks and beamstop removed. (b) The same as (a) for the sum of ten accumulated (30 s each) pulses. A non-linear logarithmic colour scale is used to display these data.
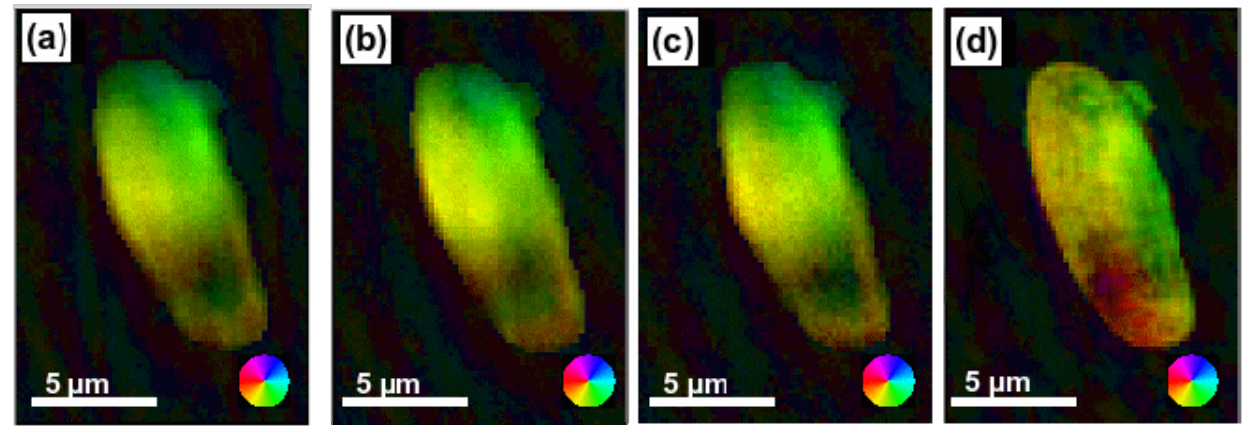

Figure 10. (Colour Online) (a-c) Reconstructed images of Navicula perminuta cell from the different single pulse diffraction patterns. (d) Same from the integrated diffraction pattern (Fig. 9(b)). In (a-d) the magnitude is encoded in the value and the phase is encoded in the hue of the image. The phase colourmap is given by the wheel in the bottom right corner of the images. Images and figures from [46].

\section{b) CXDI at the water window}

This same diatom was also measured with the third harmonic radiation of FLASH, at a wavelength of $2.66 \mathrm{~nm}$, in the so-called water window. In these measurements the incident flux was significantly less due to the smaller fraction of third harmonic radiation in the beam (about $0.5 \%$ of the total beam flux), and the reduced transmission of the beamline at these energies. Consequently an estimated $3.5 \times 10^{6}$ photons per pulse were available at the end station under these conditions. With this photon flux single pulse measurements were not feasible. We have measured integrated diffraction patterns at these energies from 10 exposures each of 30 seconds duration with FLASH delivering 30 pulses per pulse train at a $5 \mathrm{~Hz}$ pulse train repetition rate (4500 pulses per exposure). The measured diffraction pattern is shown in Fig. 11(a) scattering to a resolution of $230 \mathrm{~nm}$. Examination of this diffraction pattern reveals poorer contrast when compared with the single shot and integrated diffraction patterns produced using the fundamental radiation (Fig. 9). We attribute this reduction in 
contrast to a significantly reduced effective coherence length of the radiation at the third harmonic, which becomes smaller than the size of our diatom along its major axis.
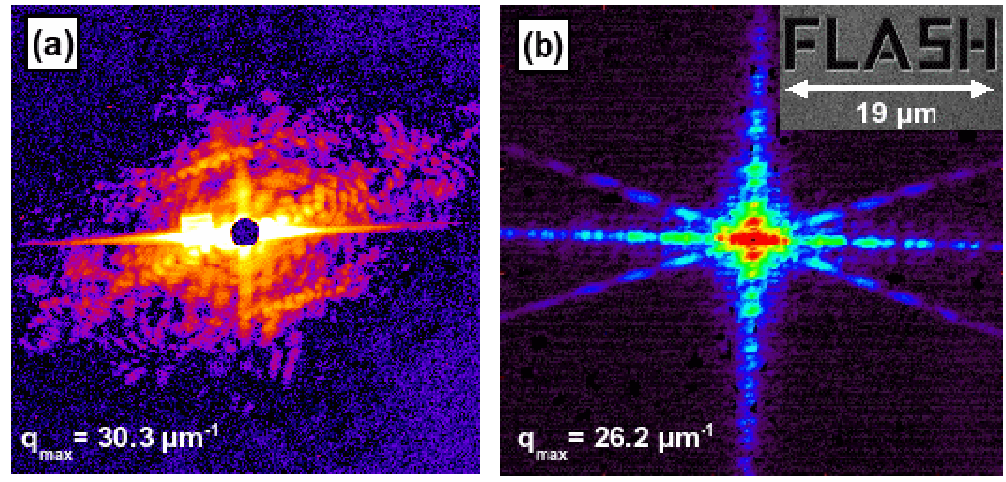

Figure 11. (Colour Online) (a) Diffraction pattern measured at the $3^{\text {rd }}$ harmonic of FLASH at $2.66 \mathrm{~nm}$ accumulated for 300 seconds. (b) Diffraction data from 'FLASH' letters. (inset) Scanning Electron Micrograph of the sample. A non-linear logarithmic colour scale is used to display these data.

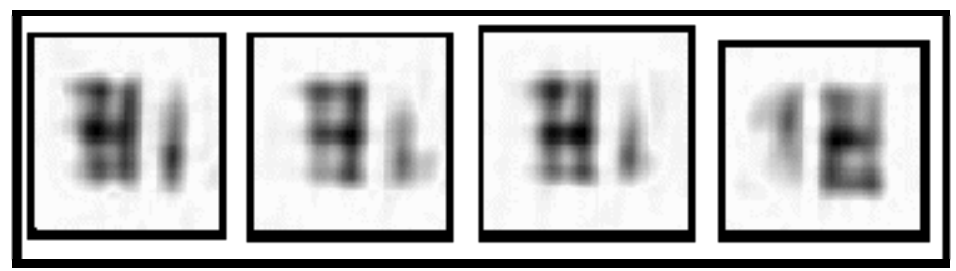

Figure 12. Different reconstructions from the $3^{\text {rd }}$ harmonic diffraction pattern shown in Fig. 11(b). Note the appearance of superimposed letters, such as ' $F$ ', ' $L$ ', ' $S$ ' and ' $H$ ' which is characteristic of a reduced coherence length. This prevents the iterative reconstruction from converging to a unique solution.

Similarly, we have illuminated a micro-fabricated series of letters spelling 'FLASH' with the third harmonic radiation, and measured integrated diffraction data scattering from that sample (see Fig. 11(b)). This data is the sum of 150 exposures, each of four seconds duration scattering to a momentum transfer that corresponds to about a $240 \mathrm{~nm}$ resolution. We see in Fig. 12 that reconstructions from this measured data fail in a characteristic way that is indicative of an insufficient effective coherence length. We see letters from the word 'FLASH' superimposed in the reconstruction, demonstrating that there is no correlation on the length scale between the different letters in the wavefield at the plane of the sample.

This lack of correlation observed in both CXDI measurements that used the $3^{\text {rd }}$ harmonic of FLASH at the PG2 beamline concords with our coherence measurements using URA apertures described in section 2(b). Indeed, the measured effective coherence length at the third harmonic (for an integrated measurement case) was approximately $5 \mu \mathrm{m}$ for these conditions that is well below the size of our sample. It is unsurprising then that our CXDI reconstructions suffer from low coherence effects. One possible way to reconstruct images under these conditions will be to characterize the FEL radiation 
and to use mode decomposition of the correlation functions (similar to that described in section 2(c)) in the reconstruction procedure [48].

\section{c) Fourier Transform Holography}

Here we report on coherent imaging of the earlier discussed Navicula sample with Fourier transform holography performed at FLASH [46]. An inverse Fourier transform was applied to the integrated CXDI measurements shown in Fig. 9(b). Unknown at the time of measurement, a point scatterer was present in the beam near the diatom. As a result, an inverse Fourier transform yields the reconstructed image of the diatom shown in Fig. 13(b) and that of a second diatom shown in Fig. 13(c). It can be seen that mostly edge information about the diatoms is reconstructed, including the 'rib'-like structure, with a clearly resolved period of $550 \mathrm{~nm}$, visible in Fig. 13(c). This edge contrast can be explained by the absence of low frequency data due to the presence of the beamstop, leaving only higher spatial frequency information to be reconstructed. It is difficult to quantify the reference-limited resolution of this reconstruction as the point scatterer had not been characterized prior to the experiment. Based on conservative resolution estimates we can conclude that the resolution here is better than $450 \mathrm{~nm}$.
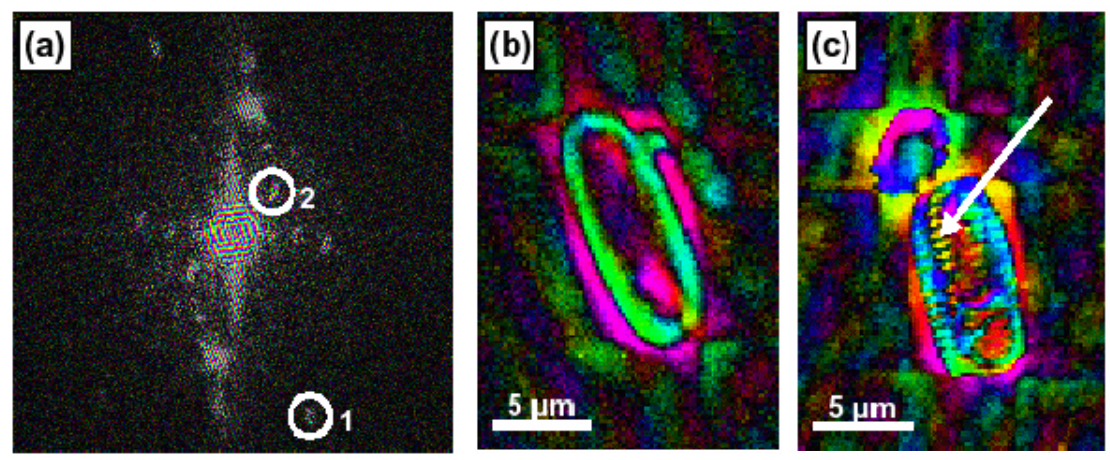

Figure 13 (Colour Online) (a) Inverse Fourier transform of the data shown in figure 9(b). Note the two FTH images of diatoms indicated by circles 1 and 2 and shown in detail in (b) and (c) respectively. (b) Reconstructed Fourier transform hologram of the diatom sample. (c) FTH reconstruction of a different cell. The colour scale represents the phase (as for the earlier reconstructions shown in figure 10).

\section{d) Analysis of achievable resolutions}

We estimate that the resolution of the reconstructed image for the integrated measurement discussed in section 4(a) was $380 \mathrm{~nm}$. In this case the reconstructed resolution is limited by the size of the detector and not by the signal-to-noise ratio. Comparing the reconstructed far-field intensities with a Modulation Transfer Function (MTF) for an incoherent imaging system [49], we estimate that if the 
detector was larger we would reconstruct an image of the diatom up to $225 \mathrm{~nm}$ resolution from this measurement. Similar calculations give a resolution of $650 \mathrm{~nm}$ for the brightest single shot images shown in Fig. 10 (a-c). In these cases the resolution is limited by the signal-to-noise ratio at high $q$ values (see Fig. 9(a)), which is clearly a function of the incident coherent flux.

It is interesting to make some predictions about the optimal resolutions achievable for our Navicula sample illuminated at $8 \mathrm{~nm}$ wavelength assuming we can deliver almost all of the $10^{13}$ photons of the FEL to the sample. The brightest measured single pulse CXDI case used approximately $1.6 \times 10^{9}$ photons incident on the sample area to produce a $650 \mathrm{~nm}$ resolution real space image of the diatom cell. The integrated case required $\sim 3.7 \times 10^{11}$ photons to deliver a possible ultimate resolution of $225 \mathrm{~nm}$. These measurements concord roughly with the expected behavior that the resolution achievable scales as the fourth power of the scattered intensity [5]. By optimizing the beamline optics to improve transmission at these energies we could expect, in principle, to have $10^{12}-10^{13}$ coherent photons per pulse on the sample area. With this available flux, using the aforementioned scaling law, a resolution of down to $60 \mathrm{~nm}^{2}$ could be expected for single pulse imaging of similar biological samples at the same experimental conditions.

\section{Conclusions and Outlook}

In this overview we have presented results of coherent diffractive imaging experiments at FLASH, the world's first user accessible XUV free-electron laser. In particular, we have shown an experimental and theoretical analysis of the coherence properties of FLASH - the key property of the radiation relevant to coherent imaging - at a fundamental wavelength of $13.7 \mathrm{~nm}$. Our analysis shows that FLASH is indeed a highly coherent source, which can be well described by a few coherent modes. We have further demonstrated that the degree of coherence is similar in both the horizontal and vertical directions, with coherence lengths of about three hundred microns at a distance $20 \mathrm{~m}$ downstream from

the source. Additionally, we have demonstrated that the measured transverse coherence length at the $3^{\text {rd }}$ harmonic of FLASH at $2.7 \mathrm{~nm}$ of a series of averaged pulses at the PG2 beamline is about $5 \mu \mathrm{m}$. The analysis of single pulses measured in the same conditions will be reported elsewhere [38]. The understanding of the coherence properties of FLASH allows the successful utilization of the FEL radiation for CXDI, and other coherence based techniques.

Our second result shown in this article was the reconstruction of a 2D crystal structure from a

\footnotetext{
${ }^{2}$ Compare to the resolution obtained in the reference [26].
} 
single pulse train of FLASH radiation. The salient points of this demonstration are the dependence of the reconstructed resolution on the signal-to-noise ratio in the data, and the improvement in signal available from 2D crystallinity. A higher degree of binning improves the signal to noise ratio and results in an improved reconstruction [45]. Additionally, we demonstrated that the traditionally noncrystalline framework of coherent diffractive imaging is applicable to two-dimensional finite crystals. For this experiment the 2D crystalline structure has been essential in providing the necessary signal to determine the structure of the unit cells. If a single unit cell was used here, simulations suggest that a successful reconstruction - to the resolution shown here - would be impossible.

Finally, we have given an overview of single-pulse, femtosecond coherent diffractive imaging for a single cellular organism (a diatom) supported by a membrane. We have verified that images reconstructed using CXDI are similar for statistically different FEL pulses. This is especially important for the success of single particle imaging of reproducible samples injected into an FEL beam and imaged by different FEL pulses. We also demonstrate here a comparison of coherent diffractive imaging and Fourier Transform Holography using FEL radiation applied to the same biological sample. Moreover, we have shown CXDI measurements using a higher harmonic of FLASH extending into the water window. From our investigation it is reasonable to expect that with future, shorter wavelength XFEL sources it will be possible to make single pulse CXDI measurements in the water window to resolutions of tens of nanometers, opening the way to biological imaging that circumvents the conventional radiation damage limit.

In summary, we have highlighted different examples of CXDI and examined the key property of coherence at FLASH. With new, hard x-ray FEL sources [50,51,52] now coming online, and the continued improvement in the interpretation and understanding of coherently scattered data, we expect to see the contribution of CXDI to structure determination using free-electron lasers grow into the future.

These results would not be possible without the invaluable help and support of many people. We would like especially to thank J. Schneider and E. Weckert for their ongoing interest and support during our FLASH experiments, our FLASH collaborative team including A. Rosenhahn, M. Grunze, C. Gutt, G. Grübel, S. Eisebitt, T. Wilhein, FLASH and the PG2 beamline team including N. Guerassimova, R. Treusch, J. Feldhaus. We are greatly indebted to the scientific and technical team at FLASH, in particular the machine operators and run coordinators, being the foundation of the successful operation and delivery of the SASE-FEL beam. 


\section{References}

1. E. L. Saldin, E. A. Schneidmiller, M. V. Yurkov, The Physics of Free Electron Lasers, SpringerVerlag, Berlin, (2000).

2. W. Ackermann et al., Nature Photonics 1, 336 - 342 (2007).

3. M.Woolfson and F. Hai-fu, Physical and non-physical methods of solving crystal structures, Cambridge University Press, Cambridge (1995).

4. D. Y. Parkinson, et al, Journal of Structural Biology 162, 380-386 (2008).

5. M. R. Howells, et al, J. Elect. Spectrosc. and Relat. Phenom. 170, 4 (2009).

6. R. Neutze, et al, Nature 406, 752 (2000).

7. K. J. Gaffney, and H. N. Chapman, Science 316, 1444 (2007).

8. J. Miao, et al., Nature 400, 342 (1999).

9. M. Pfeifer, et al., Nature 442, 63, (2006).

10. H. N. Chapman, et al., J. Opt. Soc. Am. A 23, 1179 (2006).

11. G. J. Williams, et al., Phys. Rev. Lett. 97, 025506 (2006).

12. D. Shapiro, et al., PNAS 102, 15343-15346 (2005).

13. R. H. T. Bates, Optik 61, 247 (1982).

14. J. R. Fienup, Appl. Opt. 21, 2758 (1982).

15. V. Elser, J. Opt. Soc. Am. A 20, 40 (2003).

16. S. Marchesini, Rev. Sci. Instrum. 78, 011301 (2007).

17. R. Millane, J. Opt. Soc. America A-Optics image science and vision 13, 725-734 (1996).

18. S. Lindaas, M. Howells, C. Jacobsen and A. Kalinovsky, J. Opt. Soc. Am. A 13, 1788 (1996).

19. A. Rosenhahn, R. Barth, F. Staier, et al., J. Opt. Soc. Am. A 25, 416 (2008).

20. A. Rosenhahn, et al., Opt. Express 17, 8220 (2009).

21. I. McNulty, J. Kirz, C. Jacobsen, et al., Science 256, 1009 (1992).

22. S. Eisebitt, J. Lüning, W. F. Schlotter, et al., Nature 432, 885 (2004).

23. L.-M. Stadler, C. Gutt, T. Autenrieth, et al., Phys. Rev. Lett. 100, 245503 (2008).

24. R. Treusch, and J. Feldhaus, New J. Phys. 12, 035003 (2010).

25. A. P. Mancuso, O. M. Yefanov, and I. A. Vartanyants, J. Biotechnology (2010) (published online: 16-MAR-2010. DOI: 10.1016/j.jbiotec.2010.01.024.).

26. H. N. Chapman, et al., Nature Physics 2, 839 - 843 (2006).

27. H. N. Chapman, et al., Nature 448, 676 - 679 (2007).

28. A. Barty, et al., Nature Photonics 2, 415 - 419 (2008).

29. I. A. Vartanyants, I. K. Robinson, I. McNulty, et al., J. Synchrotron Rad. 14, 453 (2007).

30. A. Singer, et al., Phys. Rev. Lett. 101, 254801 (2008).

31. J. W. Goodman, Statistical Optics, Wiley, New York (1985).

32. L. Mandel and E. Wolf, Optical coherence and quantum optics, University Press, Cambridge (1995).

33. J. J. A. Lin, D. Paterson, A. G. Peele, et al., Phys. Rev. Lett. 90, 074801 (2003).

34. I. A. Vartanyants and I. K. Robinson, J.Phys.: Condens. Matter 13, 10593-10611 (2001).

35. I. A. Vartanyants and I. K. Robinson, Optics Communications 222, 29-50 (2003).

36. K. A. Nugent and J. E. Trebes, Rev. Scient. Instr. 63, 2146-2151 (1992).

37. M. Wellhoefer, et al., J. Opt. A: Pure Appl. Opt. 9, 749 (2007).

38. A. Singer, et al., (in preparation).

39. I.A. Vartanyants, and A. Singer, New J. Phys. 12035004 (2010).

40. G. Bortel, et al., J. Struct. Biol. 166, 226-233 (2009). 
41. O.M. Yefanov, I.A. Vartanyants and E. Weckert (2010) (in preparation).

42. N. T. D. Loh and V. Elser, Phys. Rev. E, 80, 026705 (2009).

43. R. Fung, et al., Nature Physics 5, 64-67 (2009).

44. E. E. Uzgiris and R. D. Kornberg, Nature 301, 125 (1983).

45. A. P. Mancuso, et al., Phys. Rev. Lett. 102, 035502 (2009).

46. A. P. Mancuso, et al., New J. Phys. New J. Phys. 12035003 (2010).

47. C. C. Chen, J. Miao, C. W. Wang and T. K. Lee, Phys. Rev. B 76, 064113 (2007).

48. L. W. Whitehead, et al., Phys. Rev. Lett. 103, 243902 (2009).

49. J. W. Goodman, Introduction to Fourier Optics, McGraw-Hill Co, New York, (1968).

50. P. Emma (LCLS team), Proceedings of PAC 2009, H3PBI01, IEEE, Vancouver, Canada (2009).

51. T. Tanaka, T. Shintake, Eds, SCSS X-FEL Conceptual Design Report, SCSS XFEL, RIKEN, 679-5148 (2005) http://www-xfel.spring8.or.jp/.

52. M. Altarelli, et al., (Eds.), XFEL Technical Design Report, DESY 2006-097 (2005)

http://xfel.desy.de/tdr/index_eng.html. 